\title{
ATIVOS COSMÉTICOS PARA O TRATAMENTO DA LIPODISTROFIA GINÓIDE E ADIPOSIDADE LOCALIZADA
}

DOI: $10.22289 / 2446-922 X . V 3 N 2 A 10$

\author{
Kelly de Abreu Torres ${ }^{1}$ \\ Lilian de Abreu Ferreira
}

\section{RESUMO}

O tratamento tópico para a lipodistrofia, adiposidade localizada e a celulite é amplamente utilizado na estética, principalmente em associação a outros recursos terapêuticos estéticos, contribuindo na obtenção de resultados satisfatórios. Os ativos tópicos incluem agentes que aumentam o fluxo da microcirculação, que reduzem a lipogênese e promovem a lipólise, que restabelecem a estrutura normal da derme e do tecido subcutâneo, e agentes que eliminam os radicais livres ou impedem sua formação. O objetivo do estudo foi realizar uma pesquisa qualitativa e bibliográfica sobre a ação dos principais ativos utilizados no tratamento da adiposidade localizada e celulite. Para tanto, foi realizada uma busca na base de dados Pubmed sobre os ativos tópicos utilizados no tratamento da celulite e adiposidade localizada, no período de 2006 a 2016. Foram identificados alguns ativos lipolíticos como as metilxantinas, que atuam aumentando os níveis de AMPc, o retinol, que aumenta a espessura do colágeno dérmico e melhora o contorno das fibras elásticas, melhorando o aspecto da pele, e a L-carnitina, que auxilia na continuidade da lipólise. Como anticelulíticos foram encontrados - Ginkgo biloba e a Centella asiatica que atuam, basicamente, melhorando a circulação sanguínea. Apesar dos avanços na área da cosmetologia, não foram encontrados princípios ativos inovadores. As metilxantinas são predominantes no tratamento dessas duas condições. É inegável que o tratamento tópico satisfatório, em última análise, dependerá de observação e acompanhamento da condição.

Palavras-chaves: Tecido Adiposo; Lipodistrofia; Cosméticos; Celulite.

\section{ABSTRACT}

The topical treatment for localized adiposity and cellulite is widely used in aesthetics, mainly in association with other aesthetic therapeutic resources, contributing in obtaining satisfactory results. Topical compounds include microcirculation-enhancing agents that reduce lipogenesis and promote lipolysis, which restore the normal structure of the dermis and subcutaneous tissue and agents that eliminate free radicals or prevent their formation. The objective of the study was to conduct a qualitative and bibliographical research on the action of the main assets used in the treatment of localized adiposity and cellulite. For this purpose, a search was made in the Pubmed database on the active substances used in the treatment of cellulitis and

\footnotetext{
1 Endereço eletrônico de contato: abreukelly@yahoo.com.br
}

Recebido em 26/09/2017. Aprovado pelo conselho editorial para publicação em 18/10/2017.

Rev. Psicol Saúde e Debate. Dez., 2017:3(2):115-130. 
localized adiposity in the period from 2006 to 2016. Some lipolytic actives were identified, such as methylxanthines, which act to increase levels of AMPc, retinol, which increases the thickness of the dermal collagen and improves the contour of the elastic fibers, improving the appearance of the skin, and L-carnitine, which assists in the continuity of lipolysis. Ginko Biloba and Asian spark have been found to be anti-cellulitis, which basically improve blood circulation. Despite the advances in the area of aesthetics and cosmetology, no active topics were found. Methylxanthines are predominant in the treatment of these two conditions. It is undeniable that satisfactory topical treatme

nt will ultimately depend on observation and monitoring of the condition.

Keywords: Adipose tissue; Lipodystrophy; Cosmetics; Cellulite.

\section{INTRODUÇÃO}

A lipodistrofia ginóide (LG) ou celulite é caracterizada pela presença de ondulações na pele, descritas por alguns autores como aparência de casca de laranja. É o resultado de depósitos localizados de adipócitos e edemano tecido subcutâneo. Nas mulheres, os septos fibrosos de tecido conjuntivo, que segregam a gordura nos canais, estão orientados longitudinalmente, da fáscia muscular até a derme. À medida que a camada de gordura se expande, ela é projetada superficialmente, criando a aparência não uniforme da pele. Nos homens o entrecruzamento padrão do tecido conjuntivo nas coxas e glúteos que mantém a camada de gordura, evita a projeção da tecido adiposo na superfície da pele.

A adiposidade localizada consiste em um acúmulo excessivo de gordura caracterizado por uma hipertrofia das células adiposas em regiões específicas do corpo. Nos homens, possui maior predominância na região do abdômen, já nas mulheres há maior predominância em regiões glúteas, posterior e lateral da coxa (Bravo, Issa, Muniz de \& Torrado, 2013; Christensen, 2014; Hexsel \& Soirefmann, 2011).

Após a puberdade, estima-se que mais de 90\% das mulheres, possam apresentar algum grau de lipodistrofia ginóide em qualquer área onde há predominância do tecido adiposo, sendo o quadril, glúteos e membros inferiores as áreas mais suscetíveis. Em homens essa condição é extremamente rara. Embora a adiposidade ocorra em áreas com lipodistrofia ginóide, há também outras alterações estruturais na derme e microcirculação presentes que não são vistas na adiposidade. A adiposidade localizada e a lipodistrofia ginóide estão entre as principais queixas de insatisfação corporal, principalmente entre as mulheres (Bravo et al., 2013; Christensen, 2014).

A valorização da aparência torna o corpo o foco de constante necessidade de investimento, para que se atinja uma estética considerada como ideal, muito influenciada atualmente, pela exposição exagerada às mídias sociais (Brandão \& Junior, 2015; Viana \& 
Junior, 2017). Tanto a adiposidade localizada quanto a lipodistrofia ginóide possuem impacto não só na estética, mas também na qualidade de vida. Graus avançados da celulite, por exemplo, podem levar a quadros de dor, alterações das atividades funcionais, prejuízos psicológicos e à uma desagradável aparência da pele (Goldman et al., 2006).

Existem muitas modalidades de tratamento visando amenizar a aparência da LG e reduzir adiposidade localizada, como a massagem, endermologia, lipoaspiração, laser, dieta, exercício físico, entre outros (Christensen, 2014; Hexsel \& Soirefmann, 2011).

Ativos de uso tópico são amplamente utilizados, não só para se evitar procedimentos invasivos, mas também como adjuvantes a outros procedimentos, e em associação a hábitos de vida saudáveis, ajudam a delinear as formas do corpo (Bravo et al., 2013; Hexsel \& Soirefmann, 2011; Watanabe, Piazza \& Paula de, 2010; Krupek \& Costa, 2012). Na estética a aplicação dos cosméticos tem revelado resultados satisfatórios em diversas alterações da pele (Christensen, 2014; Hexsel \& Soirefmann, 2011).

Assim, o objetivo do trabalho foi realizar uma pesquisa bibliográfica integrativa sobre os ativos utilizados no tratamento da adiposidade localizada e lipodistrofia ginóide abordando os mecanismos de ação destes. Para cumprir o objetivo do estudo, foi realizada uma busca de artigos na base Pubmed. Foram selecionados artigos publicados na língua portuguesa e inglesa nos últimos 10 anos (2006-2016), que abordavam o uso de ativos tópicos para o tratamento da celulite e adiposidade localizada. Foram utilizados os termos, "cellulite", "localized adiposity" e "treatment". Não foram incluídos estudos abordando ativos tópicos em associação de outras técnicas ou procedimentos estéticos. Assim, inicialmente, para melhor compreensão, foram apresentadas as características e aspectos conceituais do tecido adiposo, da lipodistrofia ginóide e adiposidade localizada e posteriormente foram abordados os ativos tópicos encontrados nos estudos.

\section{TECIDO ADIPOSO}

A tela subcutânea ou hipoderme está situada abaixo da pele e é formada por tecido conjuntivo que pode variar do frouxo ao denso dependendo da localização, sendo formada predominantemente pelos adipócitos, células derivadas de fibroblastos. A hipoderme conecta a derme com a fáscia dos músculos subjacentes. Em mulheres de peso normal pode concentrar-se em aproximadamente de 20 a 25\% do peso corporal, sendo constituída também por tecido nervoso, fibras de colágeno, nódulos linfáticos, leucócitos, macrófagos, células do mesênquima, fibroblastos e pré-adipócitos (células precursoras dos adipócitos) (Hexsel \& 
Soirefmann, 2011; Krupek \& Costa, 2012; Lofeu, Bartolomei, Brito \& Carvalho, 2015; Azevedo, Zanin, Tolentino, Cepeda \& Busnardo, 2008).

A distribuição corporal do tecido adiposo é determinada geneticamente e é dependente de fatores como estado nutricional, como a idade, sexo e a temperatura ambiente. Há dois tipos de tecido adiposo: o amarelo ou unilocular, com células desenvolvidas, contendo uma única gotícula de gordura, que ocupa todo o citoplasma, e o tecido adiposo pardo ou multilocular, formado por células que apresentam numerosas gotículas de gordura e muitas mitocôndrias. O tecido adiposo presente nos adultos é predominantemente do tipo unilocular e apresenta septos de conjuntivo que contêm vasos e nervos. Dos septos partem fibras reticulares (colágeno III) que sustentam as células adiposas (Watanabe et al., 2010; Lofeu et al., 2015).

O tecido adiposo passou a ser considerado um verdadeiro órgão endócrino, pois além de funções como armazenamento de energia e barreira física ao trauma, os adipócitos também secretam proteínas e citocinas com capacidade de modular o estado inflamatório, denominadas adipocinas, com efeitos endócrinos, autócrinos ou parácrinos, que controlam funções como o metabolismo lipídico e glicídico, coagulação sanguínea, pressão sanguínea e de modulação hormonal (Azevedo et al., 2008; Damiani Durval \& Damiani Daniel, 2006; Junqueira \& Carneiro, 2008).

O armazenamento de energia através dos adipócitos se dá na forma de triacilgliceróis (TAG) em seu citoplasma, sem que isto seja nocivo às suas funções. Estas células apresentam seu desenvolvimento e multiplicação durante a infância e adolescência, permanecendo em número constante durante a fase adulta. Possuem todas as enzimas e proteínas reguladoras necessárias para sintetizar ácidos graxos (lipogênese) e estocar TAG em períodos em que a oferta de energia é abundante, e para mobilizá-los pela lipólise quando há restrição calórica e exercício físico. O catabolismo das reservas de gordura, denominado de lipólise, é controlado por hormônios (catecolaminas, glucagon, paratormônio, tirotropina, hormônio melanócito estimulante e adenocorticotropina), citocinas e adipocinas (Watanabe et al., 2010; Krupek \& Costa, 2012).

O sistema nervoso autônomo exerce efeito direto sobre o tecido adiposo através do sistema simpático e parassimpático. A inervação simpática referencia-se principalmente com as ações catabólicas, tais como a lipólise mediada pelos receptores $\beta$-adrenérgicos e é dependente da atividade da enzima lipase hormônio-sensível (LHS). Já o parassimpático relaciona-se com a execução de efeitos anabólicos sobre os depósitos de adipócitos, como a captação de glicose e de ácidos graxos estimulada pela insulina (Krupek \& Costa, 2012; Junqueira \& Carneiro, 2008). 
Os adipócitos possuem receptores $\beta$-adrenérgicos (agonistas) e $\alpha 2$ - adrenérgicos (antagonistas) associados à proteína G estimulatória e inibidora, respectivamente. Quando o receptor adrenérgico $\beta$ é estimulado, ocorre a ativação da enzima de membrana adenilciclase que transforma o ATP em AMPc, a proteína quinase inativa é ativada e, assim, também a triglicéride lipase que irá hidrolisar os triacilgliceróis sob a forma de ácidos graxos e glicerol. Os ácidos graxos liberados são metabolizados ou atravessam a membrana da célula e chegam à circulação sanguínea na forma de ácidos graxos não esterificados (NEFAs), sendo transportados pela albumina para as regiões que o utilizam como substrato. Já o glicerol, como é solúvel no plasma, é captado pelo fígado para sintetizar novas moléculas de glicose (Watanabe et al., 2010; Guirro \& Guirro, 2010; Fonseca-Alaniz, Takada, Alonso-Vale \& Lima, 2006).

Nos adipócitos da região glútea e coxas, há elevada expressão dos receptores $\alpha 2$ em relação aos receptores $\beta$ que estão em maior concentração na região abdominal, por isso é mais difícil a redução de medidas nos glúteos e coxas (inibem a lipólise), ao contrário da região abdominal (favorecem a lipólise) (Watanabe et al., 2010; Fonseca-Alaniz et al., 2006).

Independente da expressão dos receptores adrenérgicos, no tecido adiposo o balanço entre os dois tipos de receptores $(\alpha$ e $\beta$ ) regulará a excessiva liberação de NEFAs de alguns depósitos de gordura e será importante para a regulação da capacidade de armazenamento de TAGs e hipertrofia dos adipócitos. Quando há um mau funcionamento deste sistema, grandes quantidades de NEFAs são liberadas, resultando em alterações da sinalização insulínica e resistência insulínica (Azevedo et al., 2008).

\section{LIPODISTROFIA GINÓIDE E ADIPOSIDADE LOCALIZADA}

A adiposidade localizada consiste em uma deformação dos adipócitos caracterizada como um distúrbio no metabolismo de gordura e acúmulo de gordura em regiões específicas do corpo, como quadris, oblíquo, abdômen e coxas. O acúmulo pode variar de diferentes formas, dependendo de fatores como gênero, hormônios e genética individual. Nos homens, o abdômen é a região que possui a maior dominação de células adiposas, já nas mulheres há maior predominância em regiões glúteas, posterior e lateral da coxa (Krupek \& Costa, 2012; Silva et al.; 2014).

$\mathrm{Na}$ mulher, a localização pode ser influenciada por seu biótipo, classificada como ginóide, quando o acúmulo se localiza na metade inferior do corpo, androide, se na metade Rev. Psicol Saúde e Debate. Dez., 2017:3(2):115-130. 
superior, e mistas quando há acúmulo das ambas as regiões. Mesmo mulheres com Índice de Massa Corporal (IMC) adequado podem apresentar gordura localizada, porém a predominância é mais expressiva em mulheres com sobrepeso. A maioria das mulheres que sofrem deste distúrbio muitas vezes apresentam também problemas psicossociais, por estarem fora do padrão de beleza imposto pela sociedade (Azevedo et al., 2008; Gomes \& Damazio, 2009; Milani, João \& Farah, 2006; Machado et al.; 2009).

O processo de desenvolvimento de gordura corporal ocorre em razão do aumento no número de células adiposas, a hiperplasia celular; do aumento no volume de células já existentes, a hipertrofia celular; bem como da combinação destes dois fenômenos. Ela pode ser de origem genética ou causada por alterações posturais circulatórias onde se desenvolve a irregularidade do tecido conjuntivo adiposo subcutâneo (Guirro \& Guirro, 2010).

$\mathrm{Na}$ infância, a má formação das células adiposas é o principal motivo para a formação da gordura localizada. Porém, ao longo dos anos, a adiposidade sofre a influência de alguns fatores predisponentes, como o desequilíbrio hormonal, genética, idade e sexo. O estresse, fumo, sedentarismo, maus hábitos alimentares e disfunções estão entre alguns dos fatores determinantes que podem agravar a adiposidade (Lofeu et al., 2015; Guirro \& Guirro, 2010).

Até a adolescência, ocorre a hiperplasia dos adipócitos e, durante toda a vida, sofrem a hipertrofia, funcionando assim como reservatório de energia. Mesmo com uma alimentação equilibrada e a prática frequente de exercícios físicos, as gorduras localizadas não são totalmente mobilizadas, permanecendo no local afetado e se tornando um incômodo ponto de vista estético (Guirro \& Guirro, 2010; Borges, 2010; Cavalheiro, Ferreira \& Assunção, 2012).

A gordura é armazenada no interior dos adipóditos quando as calorias nutricionais consumidas extrapolam as dispendidas pelo organismo. Os adipócitos se caracterizam pelo acúmulo de lipídios em seu citoplasma, sob forma de pequenas gotas suspensas no citosol, e o número e tamanho dessas gotas pode variar consideravelmente. Os adipócitos da gordura localizada são mais receptivos à glicose do que as outras células de gordura e, assim, são mais resistentes à redução do peso, explicando o fato de que as dietas garantem uma redução de peso generalizada, porém, em locais onde há adiposidade localizada, a perda é mínima (Cavalheiro et al., 2012).

No corpo humano, há uma capacidade limitada para o armazenamento de carboidratos e proteínas. Dessa forma, o tecido adiposo representa um depósito de energia, principalmente em momentos de jejum prolongado, proteção contra frio ou quando o organismo está submisso à atividade intensa. Sendo assim, a gordura localizada possui suas funções limitadas no corpo, depois passa a ser prejudicial e incômoda esteticamente (Borges, 2010; Cavalheiro et al., 2012).

Rev. Psicol Saúde e Debate. Dez., 2017:3(2):115-130. 
A LG ou celulite é uma alteração da superfície da pele que se apresenta clinicamente com aspecto acolchoado, em casca de laranja ou em saco de nozes (Figura 1). Apesar de ser considerada clinicamente independente da obesidade, as portadoras de LG podem apresentar adiposidade localizada, com um aumento significativo da espessura do tecido adiposo subcutâneo, influenciando no processo da etiopatogenia (Menezes, 2006).

O colágeno desempenha um importante papel na fisiopatologia do LG. O conteúdo de colágeno muda dramaticamente à medida que os adipócitos sofrem hipertrofia ligada ao armazenamento de uma concentração elevada de triglicerídeos. Não há apenas diferenças quantitativas, mas também qualitativas na estrutura do colágeno entre o tecido gorduroso normal e hipertrófico - o último tem uma quantidade pronunciada de colágeno tipo IV e VI. Sabe-se que a ausência de colágeno VI no tecido gorduroso induz a uma expansão desregulada dos adipócitos e que a concentração desta colusão define o grau de gordura e celulite (Kruglikov, 2012).

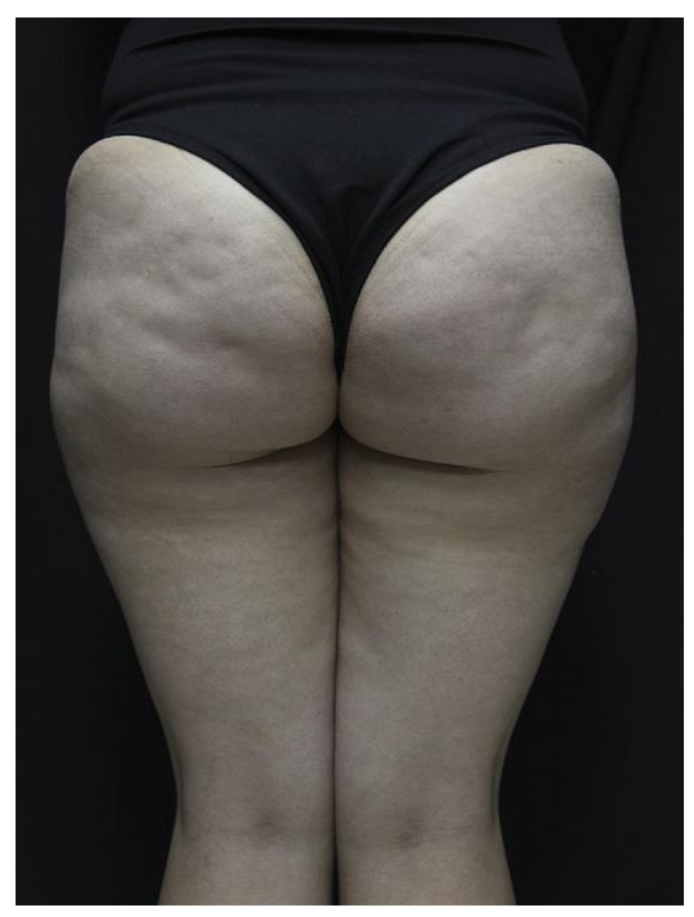

Figura 1: Aspecto clínico da lipodistrofia ginóide. Fonte: (Hexsel \& Soirefmann, 2011).

Além disso, a fibrose (aumento e desorganização de colágeno) do tecido adiposo é uma caracteristíca geral dos adipócitos metabolicamente alterados, o que é necessário para restringir sua expansão adicional. A fibrose formada pela contração das fibras do tecido conjuntivo dificulta a microcirculação e a oxigenação local, provocando a morte e a esclerose 
celular. Há uma hiperpolimerização dos mucopolissacarídeos causando a retenção de água, sódio e potássio, elevando, assim, a pressão intersticial e causando a compressão de veias, vasos linfáticos e nervos. Histologicamente há uma extrema variabilidade no tamanho e forma dos adipócitos, edema dermal, dilatação dos vasos linfáticos e aparecimento de locais com hiperqueratose, caracterizando assim os estágios da celulite (Gomes \& Damazio, 2009).

Os estágios do LG podem ser classificados em quatro tipos, do grau I ao IV. O grau I é assintomático e as irregularidades cutâneas são observadas apenas com a pressão do tecido ou com a contração muscular. Inicia-se a retenção de fluidos e, em razão ao armazenamento de lipídeos, os adipócitos começam a aumentar seu volume. No grau II observam-se pequenas alterações na superfície cutânea quando o indivíduo está na posição ortostática e sem pressão do tecido. Há comprometimento do fluxo sanguíneo em alguns locais e diminuição na elasticidade da pele. Não há alteração na sensibilidade à dor. No grau III predomina a aparência de "casca de laranja". Há redução da temperatura no local, alteração na sensibilidade e dor a palpação. No grau IV há presença de nódulos maiores palpáveis e dolorosos, pele com aparência de "saco de nozes" com formação de depressões bem mais profundas, pele flácida e enrugada em alguns pontos e em outros, compacta. Há fibrose do tecido com comprometimento nervoso (Almeida, Kilian \& Moreira, 2015; Schonvvetter, Soares \& Bagatin, 2014).

O aparecimento da celulite pode ser atribuído a alterações estruturais, inflamatórias, morfológicas e bioquímicas no tecido subcutâneo. Os hormônios como o estrogênio também influenciam a formação de celulite, estimulando a lipogênese e inibindo a lipólise, resultando na hipertrofia dos adipócitos. Esse mecanismo pode explicar parcialmente a maior prevalência dessa condição nas mulheres. Outra evidência dessa influência hormonal é o aparecimento do fibroedema gelóide na puberdade e sua exacerbação durante a gravidez e amamentação, além da relação com o uso de contraceptivos orais (Hexsel \& Soirefmann, 2011; Lupi, Semenovitch, Treu, Bottino \& Bouskela, 2007).

Também está relacionada ao aparecimento da LG, a genética, tabagismo, distribuição do tecido adiposo, sedentarismo, estresse, idade, ingestão excessiva de cafeína e bebidas alcoólicas. Embora ainda não existam dados epidemiológicos exatos, a maioria dos estudos afirma que a celulite está presente em mais de $90 \%$ das mulheres pós-púberes e, de acordo com alguns autores, dada a sua natureza ubíqua a celulite é mais apropriadamente uma característica sexual secundária do que propriamente uma doença (Bravo et al., 2013; Christensen, 2014; Almeida, Kilian \& Moreira, 2015; Luebberding, Krueger \& Sadick, 2015).

Alguns costumes alimentares também influenciam no desenvolvimento da gordura localizada e celulite, como o consumo excessivo de bebidas gasosas, pois o gás carbônico 
presente pode converter-se em ácido carbônico que, em excesso na derme e hipoderme, leva à acidificação dos tecidos, que normalmente possui pH levemente alcalino. Com esta alteração ocorre o endurecimento das fibras proteicas (colágeno e elastina). A permeabilidade e a resistência dos capilares sanguíneos se alteram, levando formação de edemas, intoxicação do tecido, falta de nutrição e oxigenação (Gomes \& Damazio, 2009).

Ainda em relação à influência dos hábitos alimentares, o consumo excessivo de sódio contribui para a retenção hídrica; já o açúcar, o álcool e a gordura estimulam a lipogênese; a deficiência de proteínas resulta em uma desorganização das fibras tecido conjuntivo e, por fim, a baixa ingestão de água e fibras dificulta o funcionamento intestinal, causando a estase circulatória (Kede \& Sabatovich, 2009).

Devido a este acúmulo de gordura localizada, recursos que facilitem o processo de lipólise são necessários na tentativa de redução, com consequente melhoria do aspecto da pele. Para que ocorra a lipólise, a enzima triglicerídeo lipase deve ser ativada para que os triacilgliceróis sejam hidrolisados (Krupek \& Costa, 2012; Borges, 2010; Duncan, Ahmadian, Jaworski, Sarkadi-Nagy \& Sul, 2007).

Como resultado, há liberação de ácidos graxos e glicerol. Os ácidos graxos, ao cair na corrente sanguínea, são transportados para as células que os utilizam como substrato energético (Cavalheiro et al., 2012).

Por este fato, os cosméticos atualmente disponíveis demonstram pouca melhora da celulite quando utilizados como único método de tratamento, e nenhum demonstrou ser capaz de levar ao seu desaparecimento por completo. Entretanto, a combinação dos ativos tópicos com outras modalidades de tratamento e a utilização deles na continuidade após algum procedimento estético de modo a complementar e manter o resultado obtido têm sido muito utilizadas, tanto na redução das circunferências corporais em relação ao tecido adiposo quanto na melhoria na aparência da pele em relação ao LG (Hexsel \& Soirefmann, 2011).

\section{ATIVOS LIPOLÍTICOS E ANTICELULÍTICOS}

\section{METILXANTINAS}

As metilxantinas como a cafeína, aminofilina, teofilina e teobromina são classificadas como $\beta$-agonistas e constituem a principal categoria bem documentada para o tratamento da celulite (Hexsel \& Soirefmann, 2011). A metilxantina mais amplamente estudada é a cafeína, utilizada em concentrações de $1 \%$ a $2 \%$. 
A cafeína é extraída dos grãos de café da Coffea arábica L. Ela atua diretamente nas células adiposas, promovendo lipólise, inibindo a fosfodiesterase, e assim aumentando os níveis de adenosina monofosfato cíclica (AMPc), que ativa a enzima lipase de triglicerídeos e os quebra em ácidos graxos livres e glicerol. A cafeína também possui efeito estimulante sobre a microcirculação cutânea (Duncan et al., 2007).

Foi demonstrado em um estudo que uma emulsão contendo cafeína aplicada por 21 dias reduziu em $17 \%$ o diâmetro das células gordurosas de ratos em comparação com o controle. Porém, a cafeína em uma formulação de gel não demonstrou alterações significativas no diâmetro das células adiposas. Os autores concluíram que o gel não é um veículo adequado para a cafeína (Velasco et al., 2008).

Uma solução contendo 7\% de caféina foi aplicada diariamente, em apenas uma perna (coxa e quadril), em mulheres de 20 a 39 anos. A outra perna foi mantida como controle. Ao final do estudo, das 134 pacientes inicialmente selecionadas, 99 completaram o protocolo. Após um mês de tratamento, houve redução estatisticamente significativa das circunferências da coxa em mais de $80 \%$ dos casos e redução da circunferência do quadril em $67,7 \%$ (Lupi et al., 2007).

A aminofilina também provoca um efeito lipolítico localizado por aumentar o $\underline{\text { AMP }}$ cíclico intracelular e inibir a fosfodiesterase. Embora exista a hipótese de que a aminofilina aplicada topicamente possa penetrar através da derme para causar lipólise, isso ainda não foi cientificamente comprovado (Ribeiro, 2010).

Alguns agonistas $\beta$-adrenérgicos como o isoproterenol, adrenalina, e agonistas $\alpha$ adrenérgicos como ioimbina, piperoxano, fentolamina e diihidroergotamina também mostraram a capacidade para causar lipólise. (Hexsel \& Soirefmann, 2011).

A teofilina possui taxa de permeação inferior à cafeína na pele, baixa solubilidade em água e efeitos secundários marcantes, entretanto o ácido teofilino acético, derivado da teofilina, é hidrossolúvel e seguro para uso tópico (Krupek \& Costa 201; Ribeiro, 2010).

\section{ÁCIDO RETINÓıco}

O ácido retinoico tópico e os derivados da vitamina $A$ são utilizados como tratamentos tópicos da celulite. O retinol aplicado topicamente, na concentração de $0,3 \%$ durante um período de seis meses ou mais, é capaz de melhorar a aparência da celulite, de acordo com um estudo publicado. Esses efeitos podem estar relacionados aos efeitos dos retinóides que aumentam a espessura do colágeno dérmico e melhoram o contorno das fibras 
elásticas. Também pode atuar como agente antiadipogênico, inibindo a diferenciação de humanos das células precursoras de adipócitos (Hexsel \& Soirefmann, 2011).

Em um ensaio clínico, o tratamento tópico de uma combinação de ácido retinóico, Furcellaria lumbricalis, Fucus vesiculosus, ácido linoleico conjugado e glaucina, um alcaloide extraído de algumas plantas vegetais, foi avaliado por um dermatologista após 8 e 12 semanas de uso. Houve melhora clínica significativa em relação ao placebo e uma diminuição significativa na espessura da camada de gordura analisadas por imagens de ultrassom (AlBader et al.; 2012).

Além disso, a combinação de retinol, cafeína e ruscogenina, um ativo derivado da planta Ruscus aculeatus, é capaz de reduzir a aparência disforme da pele e aumentar a microcirculação (Dupont et al.; 2014).

\section{L-CARNITINA}

A L-carnitina transporta o ácido graxo para o interior das mitocôndrias para que seja oxidado pela adenosina trifosfato (Krupek \& Costa, 2012; Kede \& Sabatovich, 2009; Ribeiro, 2010).

Uma mistura sinérgica incluindo cafeína, carnitina, forscolina e retinol foi relatada por Roure e colaboradores para melhorar vários parâmetros ligados à celulite. $O$ estudo em explantes de pele ex vivo mostrou que a cafeína e a forscolina estimulavam a liberação de glicerol e demonstrou pela primeira vez que o retinol e a carnitina em combinação estimularam sinergicamente a proliferação de queratinócitos, o que leva a um aumento da espessura epidérmica. O estudo clínico duplo-cego, randomizado e controlado com placebo que associou medições de circunferência em cinco partes selecionadas do corpo, medições de hidratação cutânea, bem como classificação do aspecto da pele foi realizado em 78 mulheres que aplicaram o produto ou placebo duas vezes por dia por 12 semanas consecutivas. Após 4 semanas de aplicação já foram observadas reduções significativas na circunferência do abdômen, quadris-nádegas e cintura. As melhorias afetaram todas as partes do corpo medidas após 12 semanas, incluindo o aspecto da pele. No final do estudo, oito parâmetros dos treze avaliados foram significativamente melhorados no grupo ativo e comparados ao placebo (Roure, Oddos, Rossi, Vial \& Bertin, 2011).

Rev. Psicol Saúde e Debate. Dez., 2017:3(2):115-130. 


\section{GINKO BILOBA}

O Ginkgo biloba pertence à família Ginkgoaceae e os extratos de suas folhas contêm glicosídeos, lactonas, terpenos e flavonoides, entre outros. Essas substâncias reduzem a viscosidade do sangue, inibem o fator ativador de plaquetas, aumentam a tonicidade dos vasos, levando a uma melhoria na microcirculação, além de possuírem ação antirradical livre, anti-inflamatória, acionarem o metabolismo celular e inativarem a fosfodiesterase. (Kede \& Sabatovich, 2009).

Em formulações tópicas, o extrato glicólico pode ser usado em concentrações de $5 \%$ a $10 \%$. No entanto, existem na literatura relatos de hipersensibilidade ao Ginkgo em produtos anticelulíticos, assim a concentração mais amplamente recomendada é de 1 a $3 \%$ (Hexsel \& Soirefmann, 2011).

Sparavigna e colaboradores analisaram em um ensaio randomizado controlado uma formulação tópica contendo extrato de Ginkgo biloba, um vasodilatador e escina, uma saponina extraída do Aesculus hippocastanum. Após 4 semanas de uso, aplicado 2 vezes ao dia, houve melhora nos sinais e sintomas da celulite (Sparavigna, Guglielmini, Togni, Cristoni \& Maramaldi, (2011). De acordo com Hexsel e Soirefmann (2011), todas as saponinas melhoram a drenagem venosa e linfática e são capazes de reduzir o edema.

\section{CENTELLA ASIÁTICA}

A Centella asiatica é uma planta medicinal que tem sido usada na medicina popular por centenas de anos, bem como na medicina cientificamente orientada. Os compostos ativos incluem triterpenos, como o asiacosídeo e o madecassosídeo, e seus derivados, como o ácido asiáticoe o ácido madecássico (Bylka, Znajdek-Awiżeń, Studzińska-Sroka \& Brzezińska, 2013).

A Centella asiatica acelera a integração e o metabolismo de lisina e prolina, aminoácidos de estruturação do colágeno, além de favorecer a microcirculação reduzindo edemas (24). Um estudo confirmou a influência de triterpenos de $C$. asiatica no aumento do metabolismo da lisina e da prolina. Além disso, estes compostos aumentaram a síntese de tropocolágeno e mucopolissacarídeos nos tecidos conjuntivos. Os resultados obtidos mostraram o impacto de $C$. asiatica na melhoria da nutrição dos tecidos e na estimulação vascular conjuntiva (Goldman et al., 2006). 


\section{CARRAGENANA}

Em um ensaio clínico duplo-cego controlado, cinquenta mulheres com idade entre 21 e 49 anos foram recrutadas levando em consideração o índice de massa corporal e a presença de celulite, e divididas em dois grupos de 25 indivíduos. Em cada grupo, os produtos foram aplicados na pele, usando pequenos movimentos circulares até a penetração total do produto. No primeiro grupo, um gel contendo $3 \%$ de um tipo de carragenana foi aplicado em uma coxa e o placebo foi aplicado em outra coxa (controle). No segundo grupo, uma combinação de $3 \%$ cafeína e $3 \%$ de solução carragenana em gel foi aplicado a uma coxa e gel de cafeína a 3\% foi aplicado na outra coxa. A fórmula placebo escolhida para o estudo foi um gel hidroalcoólico (75\% de água / $25 \%$ de álcool desnaturado). Os resultados mostraram que após um mês de tratamento, a circunferência das coxas foi reduzida em 0,6 cm nas voluntárias que aplicaram a fórmula contendo a solução de carragenana. Esse resultado foi significativo em comparação com a cafeína e o placebo $(P<0,001)$. Os resultados foram melhores após 2 meses de tratamento, com redução na circunferência da coxa de $0,7 \mathrm{~cm}(P$ $<0,001)$. A aparência de celulite foi significativamente reduzida em $9 \%$ em comparação ao placebo e ao gel de cafeína nas voluntárias que usaram a fórmula contendo a carragenana $(P<0,05)$. Da mesma forma, e ainda em comparação com a cafeína, a aparência de celulite foi significante reduzida em $11 \%$ após 2 meses. Além disso, os dois resultados foram significativos versus o placebo $(P<0,05)$. Os autores concluíram que esse tipo de carragenana pode ser promissor no tratamento da celulite e adiposidade, e que em conjunto com a caféina pode promover ainda mais a eficácia da formulação (Vogelgesang, Bonnet, Godard, Sohm \& Perrier, 2011).

\section{CONSIDERAÇÕES FINAIS}

Apesar dos avanços na área da cosmetologia, não foram encontrados ativos tópicos inovadores. Ainda prevalecem as metilxantinas para tratar a adiposidade localizada e a celulite, sendo a cafeína o ativo com maior número de citações nos estudos.

A celulite é uma doença multifatorial que requer uma abordagem multidisciplinar, assim como a lipodistrofia localizada, para o tratamento, e isto é a razão pela qual ainda é difícil indicar um tratamento único efetivo para esta condição. As formulações tópicas possuem efeitos limitados e são mais amplamente recomendadas como um tratamento 
adjuvante. O tratamento tópico bem sucedido da celulite e gordura localizada, em última análise, dependerá de observação e acompanhamento.

\section{REFERÊNCIAS}

Al-Bader, T., Byrne, A., Gillbro, J., Mitarotonda, A., Metois, A., Vial, F., Rawlings, A.V., \& Laloeuf, A. (2012). Effect of cosmetic ingredients as anticellulite agents: synergistic action of actives with in vitro and in vivo efficacy. J Cosmet Dermatol, 11, 17-26.

Almeida, T.P. de., Kilian, T., \& Moreira, J.A.R. (2015). Comparação entre a endermoterapia e o ultrassom no tratamento do fibro edema geloide: Revista Científica da FHO/UNIARARAS, $3(1)$.

Azevedo, C.J.D., Zanin, E.C., Tolentino, T.M., Cepeda, C.C., \& Busnardo, V.L. (2008). Estudos Comparativos Dos Efeitos Da Eletrolipólise Por Acupontos e Da Eletrolipólise Por Acupontos Associada Ao Trabalho Aeróbico No Tratamento Da Adiposidade Abdominal Grau I Em Indivíduos Do Sexo Feminino Com Idade Entre 18 E 25 Anos. RUBS, 1(2), 64-71.

Borges, F.S. (2010). Modalidades terapêuticas nas disfunções estéticas. São Paulo: Phorte.

Brandão, F., \& Júnior, G. (2015). O USO DE SUBSTÂNCIAS NOCIVAS ASSOCIADAS AO COMPORTAMENTO DE RISCO DO PRATICANTE DE ATIVIDADE FÍSICA. Psicologia E SaúDe Em Debate, 1(1), 53-64.

Bravo, F.S.B., Issa, A.C.M., Muniz, S.L.R. de., \& Torrado, M.C. (2013). Tratamento da lipodistrofia ginóide com radiofrequência unipolar: avaliação clínica, laboratorial e ultrassonográfica. Surg Cosmet Dermatol, 5(2), 138-44.

Bylka, W., Znajdek-Awiżeń, P., Studzińska-Sroka., \& Brzezińska, M. (2013). Centella asiatica in cosmetology. Advances in Dermatology and Allergology/Postępy Dermatologii I Alergologii, 30(1), 46-49.

Cavalheiro, C.M., Ferreira, A.S., Assunção, F.F.O. (2012). O uso da eletrolipólise no tratamento da adiposidade localizada. Revisão integrativa Ensaios e Ciência: Ciências Biológicas, Agrárias e da Saúde 2012, 16(2).

Christensen, M.S. (2014). A successful topical therapy for cellulite. Surg Cosmet Dermatol, 6(4), 349-53.

Damiani, Durval., \& Damiani, Daniel. (2006). Tecido Adiposo: "depósito de gordura" ou um complexo órgão endócrino? Pediatr.mod, 42(2),55-56.

Duncan, R.E., Ahmadian, M., Jaworski, K., Sarkadi-Nagy, E., \& Sul, H.S. (2007). Regulation of lipolysis in adipocytes. Annu Rev Nutri, 27, 79-101.

Dupont, E., Journet, M., Oula, M.L., Gomez, J., Léveillé, C., Loing, E., \& Bilodeau, D. (2014). An integral topical gel for cellulite reduction: results from a double-blind, randomized, placebo-controlled evaluation of efficacy. Clin Cosmet Investig Dermatol, 773-788.

Rev. Psicol Saúde e Debate. Dez., 2017:3(2):115-130. 
Fonseca-Alaniz, M.H., Takada, J., Alonso-Vale, M.I.C., \& Lima, F.B.O. (2006). Tecido Adiposo Como Centro Regulador do Metabolismo. Arq Bras Endocrinol Metab, 50(2).

Goldman, M.P., Bacci, P.A., Leibaschoff, G., HexSel, D., \& Angelini, F. (2006). Cellulite: pathophysiology and treatment. Taylor \& Francis, New York, London.

Gomes, R.K., \& Damazio, M.G. (2009). Cosmetologia: Descomplicando os princípios ativos. São Paulo: Livraria Médica Paulista.

Guirro, E.C.O., \& Guirro, R.R.J. (2010). Fisioterapia dermato-funcional: fundamentos, recursos, patologias. Barueri: Manole.

Hexsel, D., \& Soirefmann, M. (2011). Cosmeceuticals for cellulite. Semin Cutan Med Surg, $30(3), 167-70$

Junqueira, L.C.U., \& Carneiro, J. (2008). Histologia Básica. Rio de Janeiro: Guanabara Koogan.

Kede, M., \& Sabatovich, O. (2009). Dermatologia Estética. São Paulo: Atheneu.

Khan, M.H., Victor, F., Rao, B., \& Sadick, N.S. (2010). Treatment of cellulite: Part I. Pathophisiology. Am Acad Dermatol, 62, 361-370.

Kruglikov, I. (2012). The pathophysiology of cellulite: can the puzzle eventually be solved? J Cosmet Dermatol Sci Appl, 2(1),1-7.

Krupek, T., \& Costa, C.E.M. da. (2012). Mecanismo de Ação de Compostos Utilizados na Cosméticas para o Tratamento da Gordura Localizada e da Celulite. Revista Saúde e Pesquisa, 5(3).

Lofeu, G.M., Bartolomei, K., Brito, L.R.A., \& Carvalho, A.A. (2015). Atuação da Radiofrequência na Gordura Localizada no Abdômem. Revista da Universidade Vale do Rio Verde, Três Corações, 13(1), 571-81.

Luebberding, S., Krueger, N., \& Sadick, N.S. (2015). Cellulite: an evidence-based review. Am J Clin Dermatol, 16(4), 243-56.

Lupi, O., Semenovitch, I.J., Treu, C., Bottino, D., \& Bouskela, E. (2007). Evaluation of the effects of caffeine in the microcirculation and edema on thighs and buttocks using the orthogonal polarization spectral imaging and clinical parameters. J Cosmet Dermatol, 6(2), $102-7$.

Machado, A.F.P., Tacani, R.E., Schwartz, J., Liebano, R.E., Ramos, J.L.A., \& Frare, T. (2009). Incidência de fibro edema geloide em mulheres caucasianas jovens. Arq Bras Ciên Saúde, 34(2), 80-6.

Menezes, J.A. (2006). Ditadura da beleza. Revista epistemo-somática, 3(2),265-267.

Milani, G.B., João, S.M.A., \& Farah, E.A. (2006). Fundamentos da fisioterapia dermato funcional: revisão de literatura. Revista fisioterapia e pesquisa,13(1), 37-43.

Ribeiro, C. (2010). Cosmetologia aplicada à dermoestética. São Paulo: Pharmabooks. 
Roure, R., Oddos, T., Rossi, A., Vial, F., \& Bertin, C. (2011). Evaluation of the efficacy of a topical cosmetic slimming product combining tetrahydroxypropyl ethylenediamine, caffeine, carnitine, forskolin and retinol, In vitro, ex vivo and in vivo studies. Int J Cosmet Sci, 33(6), 18.

Schonvvetter, B., Soares, J.L.M., \& Bagatin, E. (2014). Longitudinal evaluation of manual lymphatic drainage for the treatment of gynoid lipodystrophy. An. Bras. Dermatol, 89(5), 712718.

Silva, R.M.V., Meyer, P.F., Souza, J.F., Féliz, J.L.O., Delgado, E.B.S., Queiroz, L.O., \& Medeiros, M.L. (2014). Efeitos da fonoforese com gel de ativos lipolíticos na adiposidade abdominal. O Mundo da Saúde, 28(4), 369-74.

Sparavigna, A., Guglielmini, G., Togni, S., Cristoni, A., \& Maramaldi, G. (2011). Evaluation of anti-cellulite efficacy: a topical cosmetic treatment for cellulite blemishes - a multifunctional formulation. J Cosmet Sci, 62, 305-16.

Velasco, M.V., Tano, C.T., Machado-Santelli, G.M., Consiglieri, V.O., Kaneko, T.M., \& Baby, A.R. (2008). .Effects of caffeine and siloxanetriol alginate caffeine, as anticellulite agents, on fatty tissue:Histological evaluation. J Cosmet Dermatol, 7, 23-29.

Viana, A., \& Junior, G. (2017). QUALIDADE DE VIDA EM IDOSOS PRATICANTES DE ATIVIDADES FÍSICAS. Psicologia E Saúde Em Debate, 3(1), 87-98.

Vogelgesang, B., Bonnet, I., Godard, N., Sohm, B., \& Perrier, E. (2011). In vitro and in vivo efficacy of sulfo-carrabiose, a sugar-based cosmetic ingredient with anti-cellulite properties. Int J Cosmet Sci, 33(2), 120-5.

Watanabe, E., Piazza, F.C.P., \& Paula, V.B. de. (2010). Tratamentos para gordura localizada: uma análise dos recursos da cosmetologia e estética. Trabalho de Conclusão de Curso (Graduação em Tecnologia em Cosmetologia e Estética da Universidade do Vale do Itajaí. [Acesso em 25 nov 2016]. Disponível em: http://siaibib01.univali.br/pdf/Luize\%20Guimar\%C3\%A3es\%20de\%20Geus,\%20Milena\%20F lora\%20da\%20Silva.pdf. 